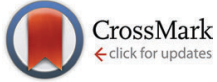

Cite this: New J. Chem., 2015, 39, 4227

Received (in Montpellier, France) 3rd February 2015,

Accepted 16th April 2015

DOI: 10.1039/c5nj00294j

www.rsc.org/njc

\title{
Silver sputtering into a liquid matrix containing mercaptans: the systematic size control of silver nanoparticles in single nanometer-orders $\dagger$
}

\author{
Yohei Ishida, Ryota Nakabayashi, Masaki Matsubara and Tetsu Yonezawa*
}

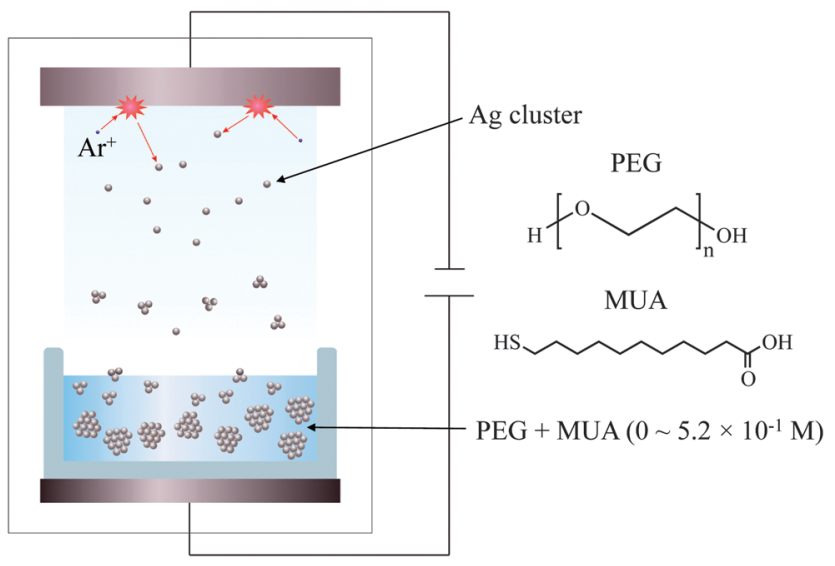

Fig. 1 Schematic illustration of the matrix sputtering method and the chemical structures of PEG and MUA.

films onto solid substrates. In our matrix sputtering process, argon is ionized by high voltage and then attacks the target metal and ejects the target atoms or clusters in a vacuum chamber. ${ }^{17}$ Ejected atoms and clusters aggregate not only in a gas phase but also inside or at the interface of the capturing liquid matrix, and form NPs.

So far, several capturing matrices have been examined for the preparation of Au NPs by the matrix sputtering method and they can be categorized into (i) 6-mercaptohexyltrimethylammoniumbromide (6-MTAB) ${ }^{15}$ and pentaerythritol tetrakis(3-mercaptopropionate) (PEMP), ${ }^{16}$ and (ii) propane-1,2,3-triol, ${ }^{18}$ polyethylene glycol (PEG), ${ }^{17,19,20}$ ionic liquids, ${ }^{21,22}$ and pentaerythritol ethoxylate (PEEL). ${ }^{16}$ Group (i) depends on the affinity of the mercapto group and can form very small $(\sim 1.3 \mathrm{~nm})$ fluorescent Au NPs, while group (ii) depends mainly on the viscosity of themselves and forms rather large Au NPs (2-5 nm). The difference in their size can be explained by the coordination of mercapto groups that can prevent the aggregation and growth of Au NPs inside or at the interface of the capture 
media. From these results, it is expected that we can synthesize metal NPs with various sizes in single nanometer-order and thus can control the physical properties of NPs by the matrix sputtering method.

While most results were for Au NPs due to its high stability and lots of previous studies, it is difficult to find other metal NPs such as silver by this matrix sputtering method except for a few reports. ${ }^{18,23}$ In this paper, we propose novel methodology to control the size of Ag NPs by the matrix sputtering method. While previous approaches have used only the capturing liquid matrix, our new approach in this study uses both the capturing matrix and organic molecules with mercapto groups. The mercaptans can dissolve in capture media and prevent the aggregation and growth of Ag NPs inside or at the interface of the capturing matrix according to the concentration of mercaptans. As far as we know, this is the first example for the systematic size control of Ag NPs by sputtering techniques.

The capture media used here was polyethylene glycol (PEG) with an average molecular weight of $600 \mathrm{~g} \mathrm{~mol}^{-1}$ and the degree of polymerization is between 10 and 17. Since PEG does not have any functional groups stabilizing metal NPs, it has been reported that the viscosity affects the size of resultant NPs. ${ }^{17}$ Thus, in the current work the amount of PEG was constant under different thiol concentrations. The thiol stabilizer used here was 11-mercaptoundecanoic acid (MUA). The experimental procedure is described below (Fig. 1). At first, PEG and MUA were dried under vacuum (rotary oil pump) at $100{ }^{\circ} \mathrm{C}$ for $2 \mathrm{~h}$ to remove volatile substances including water in order to avoid boiling inside the vacuum sputtering chamber. $10 \mathrm{~g}$ of PEG and the corresponding amount of MUA were put in a glass petri dish with a diameter of $6.5 \mathrm{~cm}$ and was horizontally set against the sputtering target. The amount of MUA in PEG was set at $0,1,5,10,100,1000 \mathrm{mg}$ (corresponding to $0,5.2 \times 10^{-4}, 2.6 \times$ $10^{-3}, 5.2 \times 10^{-3}, 5.2 \times 10^{-2}$ and $5.2 \times 10^{-1} \mathrm{M}$, respectively) and MUA completely dissolved in PEG under all conditions. Ag was sputtered by a current of $30 \mathrm{~mA}$ under Ar at a pressure of 2.0 Pa. The distance between the surface of PEG and the surface of the $\mathrm{Ag}$ target was set at $50 \mathrm{~mm}$. Sputtering was carried out for $20 \mathrm{~min}$ at room temperature under stirring at $100 \mathrm{rpm}$. Further experimental details are described in the ESI. $\dagger$

The extinction spectra of Ag NP suspension in PEG were measured in a quartz cell with $1 \mathrm{~mm}$ optical path immediately after the sputtering preparation without further purification or dilution (Fig. 2). Broad absorption peaks at around $440 \mathrm{~nm}$ $(2.82 \mathrm{eV})$, which correspond to the localized surface plasmon absorption of Ag NPs, were observed. The intensity of plasmon absorption decreased as the concentration of MUA increased, and it disappeared in $\mathrm{Ag}$ NPs prepared at $5.2 \times 10^{-2}$ and $5.2 \times$ $10^{-1} \mathrm{M}$ of MUA. Moreover, a new absorption shoulder was observed at $340 \mathrm{~nm}$ (3.65 eV) corresponding to the decrease of plasmon absorption. This absorption at $340 \mathrm{~nm}$ would be the absorption of very small Ag NPs. A similar absorption characteristic has been reported for glutathione stabilized $\mathrm{Ag} \mathrm{NPs}^{24}$ with ca. $2 \mathrm{~nm}$ diameter (at $3.40 \mathrm{eV}$ ). Surface plasmon absorption is generated by the vibration of the group of free electrons in the surface region of NPs and they show the absorption peak

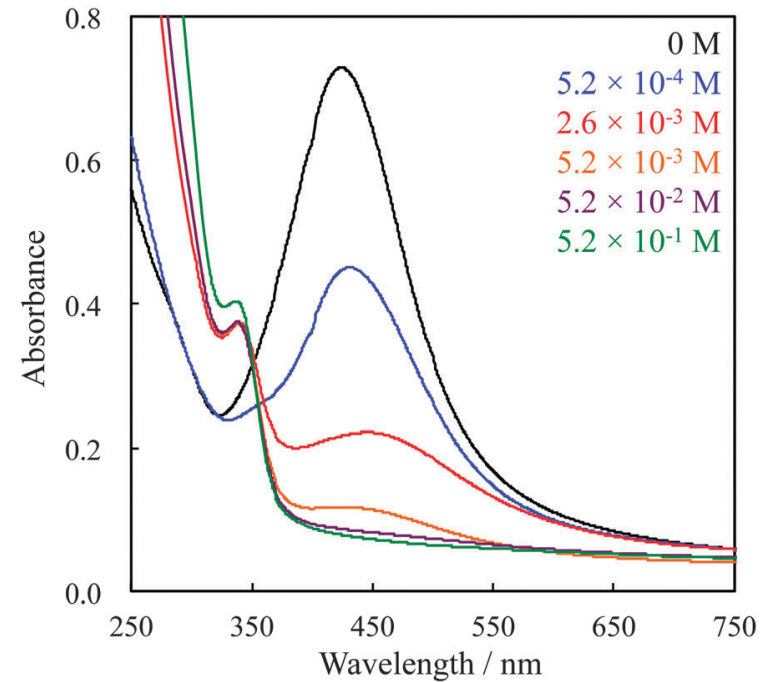

Fig. 2 Extinction spectra of Ag NPs prepared at various concentrations of MUA from 0 to $5.2 \times 10^{-1} \mathrm{M}$.

corresponding to the vibration frequency. Therefore, the generation of plasmon absorption requires a certain particle size containing certain number of atoms. ${ }^{25,26}$ When the number of atoms in each particle decreases, the energy band gap becomes wider according to the quantum size effect and the particle becomes non-metallic. Then, plasmon absorption is not observable for such small particles. Judging from the absorption spectra in Fig. 2, the size of Ag NPs would decrease by the increase of MUA concentration, and those prepared with $5.2 \times 10^{-2}$ and $5.2 \times$ $10^{-1} \mathrm{M}$ of MUA should be very small and thus no plasmon absorption was observed. The differences in their absorptions thus should be understood by particle diameters by transmission electron microscopy (TEM).

Fig. 3 shows representative TEM images and size-distribution histograms obtained from these images of Ag NPs prepared at (a) 0 , (b) $5.2 \times 10^{-3}$ and (c) $5.2 \times 10^{-1} \mathrm{M}$ concentrations of MUA (see other samples in Fig. S1, ESI $\dagger$ ). The TEM image of Ag NPs prepared without MUA (Fig. 3a) showed poly-dispersed particles, and the shape of the histogram is rather different from the Gaussian distribution. Its average diameter is $7.4 \pm 3.6 \mathrm{~nm}$. Judging from this result, it is apparent that the preparation only by PEG without MUA cannot form the regularly ordered Ag NPs. Since the NP formation in PEG depends only on the viscosity of PEG, the aggregation of NPs in PEG solution is not suppressed. Thus, the induction of MUA, which can stabilize the Ag NPs by the coordination of its thiol group, is expected to control both the size and the distribution of Ag NPs in this method. With the induction of MUA, the average sizes are $3.0 \pm 0.6$ and $2.2 \pm 0.5 \mathrm{~nm}$ for $5.2 \times 10^{-3}$ and $5.2 \times 10^{-1} \mathrm{M}$ concentrations of MUA, respectively. Most importantly, the size of Ag NPs drastically decreased and the distribution histogram became symmetrical, compared to that of Fig. 3a.

The change in the particle size as a function of MUA concentration is shown in Fig. 3d. Judging from the results, it is obvious that higher MUA concentration makes smaller Ag NPs. This tendency can be simply explained by the collision probability of MUA to Ag NPs inside and at the interface of PEG. 

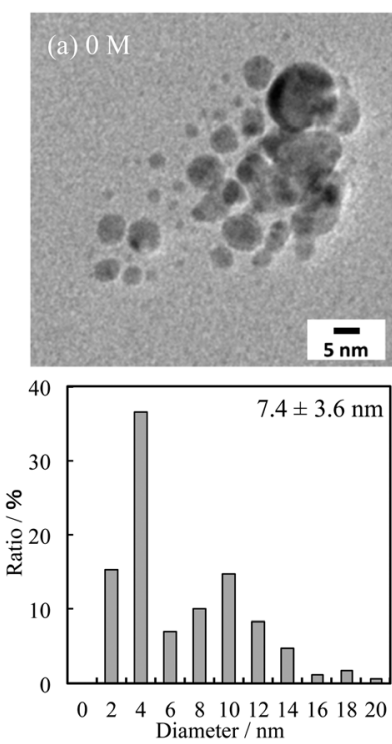
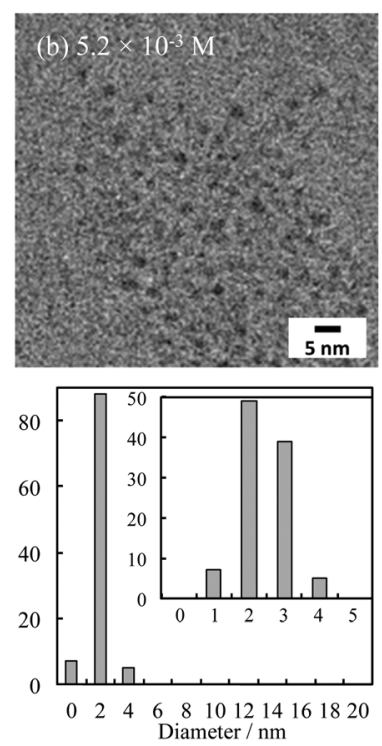
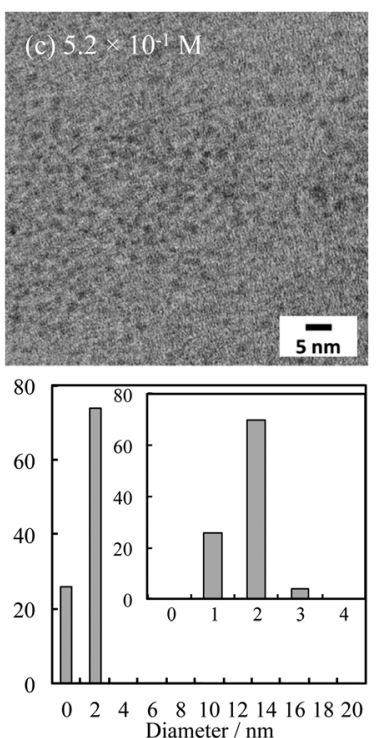

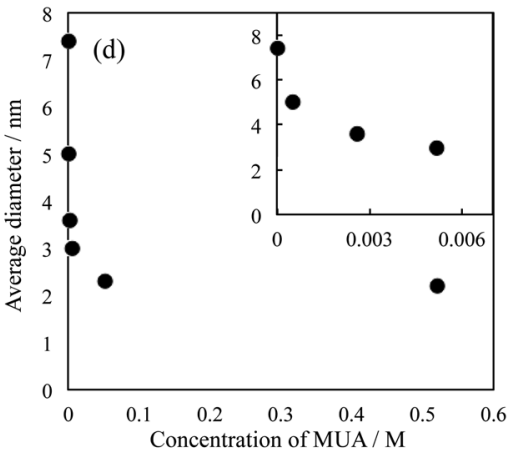

Diameter $/ \mathrm{nm}$

Fig. 3 TEM images and size-distribution histograms of Ag NPs prepared at (a) 0, (b) $5.2 \times 10^{-3}$ and (c) $5.2 \times 10^{-1}$ M concentration of MUA in PEG. The inset shows the expansion of histograms. (d) Average diameter of Ag NPs prepared at various concentrations of MUA from 0 to $5.2 \times 10^{-1} \mathrm{M}$. The inset shows the expansion plots from 0 to $0.007 \mathrm{M}$.

Hence, the decrease in their particle sizes reflects the decrease of plasmon absorption in Fig. 2. It is known that UV-Vis spectroscopic method has been used as a quantitative means to measure the concentration of particles in one size. If the particle size changes, the peak position will shift, too. Thus, we consider that the decrease of plasmon absorption in the current experiment directly reflected the decrease of the number of particles that show plasmon absorption. As a result, the diameter reached to a "cluster region" and thus we observed a new absorption shoulder at around $340 \mathrm{~nm}$ that corresponds to $\mathrm{Ag}$ clusters. This result clearly suggests that the size of Ag NPs can be controlled by the concentration of the thiol-stabilizer in the capture media, as we expected.

To clarify whether the MUA actually attaches to the surface of Ag NPs, HAADF-STEM and EDX mapping observation were carried out (see the sample preparation method in the ESI $\dagger$ ). Fig. 4 shows the HAADF-STEM image of purified Ag NPs (a), and EDX mapping images for $\mathrm{Ag}(\mathrm{b}), \mathrm{S}(\mathrm{c})$ and $\mathrm{Ag}+\mathrm{S}$ (d) in the green square area in Fig. 4a. As can be seen from the EDX mapping, the $\mathrm{S}$ atoms were obviously concentrated at the surface of $\mathrm{Ag}$ NPs. These STEM images clearly indicate the stabilization of Ag NPs by MUA.

In conclusion, we proposed a novel and easy synthetic method for size-controlled silver nanoparticles (Ag NPs) by the matrix sputtering method. While previous studies on the sputtering method have used only the capturing liquid matrix, our new approach in this study uses both the capturing matrix (PEG) and organic molecules with mercapto groups (MUA). The control of MUA concentration in PEG directly controlled the size of resulting Ag NPs from 2.2 to $7.4 \mathrm{~nm}$ in diameter. According to their size, the plasmon absorption was observed at $440 \mathrm{~nm}$ in larger NPs $(>3.0 \pm 0.6 \mathrm{~nm})$ prepared at 0 to $5.2 \times$ $10^{-3} \mathrm{M}$ of MUA, however, it was not observed and new absorption was detected at $340 \mathrm{~nm}$ for smaller NPs $(<2.4 \pm 0.5 \mathrm{~nm})$. The stabilization of Ag NPs by MUA was clarified by STEM-EDX mapping. We believe that the results presented in this paper will contribute as a novel and simple method for the preparation of functional metal NPs.
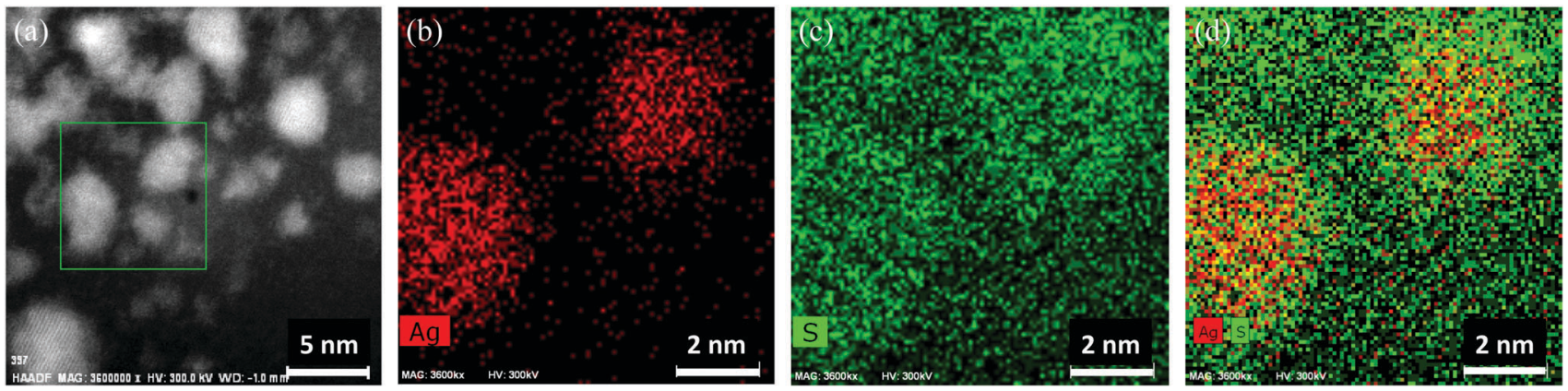

Fig. 4 HAADF-STEM image of Ag NPs prepared under $5.2 \times 10^{-1} \mathrm{M}$ concentration of MUA in PEG (a), EDX mapping images of Ag (b) and S (c) and Ag $+\mathrm{S}$ (d). The green square in image (a) indicates the area for EDX mapping for (b)-(d). 


\section{Experimental section}

Polyethylene glycol (PEG, Ave $M_{\mathrm{w}}=600$ ) was purchased from Wako. 11-Mercaptoundecanoic acid (MUA) was from SigmaAldrich. The Ag target (5 $\mathrm{mm} \varphi, 99.9 \%$ ) was supplied by Tanaka Precious Metals (Japan). Ag was sputtered by a current of $30 \mathrm{~mA}$ under $\mathrm{Ar}$ at a pressure of 2.0 Pa. The distance between the surface of PEG and the surface of the Ag target was set at $50 \mathrm{~mm}$. Sputtering was carried out for $20 \mathrm{~min}$ at room temperature under stirring at $100 \mathrm{rpm}$.

\section{Acknowledgements}

This work was partly supported by a Research Fund from Hokkaido University to YI and TY, and by Grant-in-Aid for Scientific Research (A) from JSPS and (24241041) Grant-in-Aid for Scientific Research in Priority Area "New Polymeric Materials Based on Element-Blocks (2401)" from MEXT, Japan, to TY (25102501), and by Building of Consortia for the Development of Human Resources in Science and Technology of Hokkaido University (to YI). A part of this work was also supported by Nanotechnology Platform Program (Characterization and Fabrication, Hokkaido University) of MEXT, Japan.

\section{Notes and references}

1 D. V. Talapin, J.-S. Lee, M. V. Kovalenko and E. V. Shevchenko, Chem. Rev., 2010, 110, 389.

2 F. X. Redl, K.-S. Cho, C. B. Murray and S. O'Brien, Nature, 2003, 423, 968.

3 M. Brust, M. Walker, D. Bethell, D. J. Schiffrin and R. Whyman, J. Chem. Soc., Chem. Commun., 1994, 801.

4 C. J. Kiely, J. Fink, M. Brust, D. Bethell and D. J. Schiffrin, Nature, 1998, 396, 444.

5 T. Tsukuda, Bull. Chem. Soc. Jpn., 2012, 85, 151.

6 N. Toshima and T. Yonezawa, New J. Chem., 1998, 22, 1179.

7 A. Taketoshi and M. Haruta, Chem. Lett., 2014, 43, 380.

8 H. C. Choi, S. Kundaria, D. Wang, A. Javey, Q. Wang, M. Rolandi and H. Dai, Nano Lett., 2003, 3, 157.
9 Z. Wu and R. Jin, Nano Lett., 2010, 10, 2568.

10 S. Kang, M. Yasuda, H. Miyasaka, H. Hayashi, M. Kawasaki, T. Umeyama, Y. Matano, K. Yoshida, S. Isoda and H. Imahori, ChemSusChem, 2008, 1, 254.

11 E. C. Dreaden, A. M. Alkilany, X. Huang, C. J. Murphy and M. A. El-Sayed, Chem. Soc. Rev., 2012, 41, 2740.

12 R. R. Naik, S. J. Stringer, G. Agarwal, S. E. Jones and M. O. Stone, Nat. Mater., 2002, 1, 169.

13 P. Raveendran, J. Fu and S. L. Wallen, J. Am. Chem. Soc., 2003, 125, 13940.

14 A. Kumar, P. K. Vemula, P. M. Ajayan and G. John, Nat. Mater., 2008, 7, 236.

15 Y. Shishino, T. Yonezawa, K. Kawai and H. Nishihara, Chem. Commun., 2010, 46, 7211.

16 Y. Shishino, T. Yonezawa, S. Udagawa, K. Hase and H. Nishihara, Angew. Chem., Int. Ed., 2010, 50, 703.

17 Y. Hatakeyama, T. Morita, S. Takahashi, K. Onishi and K. Nishikawa, J. Phys. Chem. C, 2011, 115, 3279.

18 J. Siegel, O. Kvítek, P. Ulbrich, Z. Kolská, P. Slepička and V. Švorčík, Mater. Lett., 2012, 89, 47.

19 H. Wender, R. V. Gonçalves, A. F. Feil, P. Migowski, F. S. Poletto, A. R. Pohlmann, J. Dupont and S. R. Teixeira, J. Phys. Chem. C, 2011, 115, 16362.

20 T. Sumi, S. Motono, Y. Ishida, N. Shirahata and T. Yonezawa, Langmuir, 2015, 31, 4323.

21 T. Torimoto, T. Tsuda, K.-I. Okazaki and S. Kuwabata, Adv. Mater., 2010, 22, 1196.

22 T. Torimoto, K.-I. Okazaki, T. Kiyama, K. Hirahara, N. Tanaka and S. Kuwabata, Appl. Phys. Lett., 2006, 89, 243117.

23 K.-I. Okazaki, T. Kiyama, K. Hirahara, N. Tanaka, S. Kuwabata and T. Torimoto, Chem. Commun., 2008, 691.

24 X. Le Guével, C. Spies, N. Daum, G. Jung and M. Schneider, Nano Res., 2012, 5, 379.

25 N. Schaeffer, B. Tan, C. Dickinson, M. J. Rosseinsky, A. Laromaine, D. W. McComb, M. M. Stevens, Y. Wang, L. Petit, C. Barentin, D. G. Spiller, A. I. Cooper and R. Lévy, Chem. Commun., 2008, 3986.

26 S. H. Yau, O. Varnavski and T. Goodson, Acc. Chem. Res., 2013, 46, 1506. 